\title{
Simultaneous enantioseparation of antiparkinsonian medication Rotigotine and related chiral impurities by capillary zone electrophoresis using dual cyclodextrin system
}

\author{
Bao-Lin Chu ${ }^{\text {a,c }}$, Baoyuan Guo ${ }^{b}$, Hongjian Zuo ${ }^{\text {a }}$, \\ Zhihua Wang ${ }^{\mathrm{a}, *}$, Jin-Ming Lin ${ }^{\mathrm{b}, \mathrm{c}, * *}$ \\ a State Key Laboratory of Chemical Resource Engineering, College of Science, \\ Beijing University of Chemical Technology, Beijing 100029, China \\ b State Key Laboratory of Environmental and Ecotoxicology, Research Center for Eco-Environmental Sciences, \\ Chinese Academy of Sciences, P.O. Box 2871, Beijing 100085, China \\ c Department of Chemistry, Tsinghua University, Beijing 100084, China
}

Received 1 January 2007; received in revised form 5 February 2007; accepted 8 February 2007

Available online 13 February 2007

\begin{abstract}
A dual cyclodextrin (CD) system consisting of sulfated $\beta-C D(S-\beta-C D)$ and methyl- $\beta-C D(M-\beta-C D)$ modified capillary zone electrophoresis (CZE) method was proposed to separate the antiparkinsonian drug Rotigotine $((-)-(S)-2$ - $(N$-propyl- $N$-2-thienylethylamino)-5-hydroxytetralin) and related chiral impurities (2-( $N$-propylamino)-5-hydroxytetralin, 2-(N-propylamino)-5-methoxytetralin). The method was optimized by varying the $\mathrm{CD}$ type, the buffer $\mathrm{pH}$, individual $\mathrm{CD}$ concentration of the dual system and the ionic strength of background electrolyte. Under the optimum conditions, i.e. $2 \%(\mathrm{w} / \mathrm{v}) \mathrm{S}-\beta-\mathrm{CD}$ and $2 \%(\mathrm{w} / \mathrm{v}) \mathrm{M}-\beta-\mathrm{CD}$ in $100 \mathrm{mM}$ sodium phosphate $(\mathrm{pH} 2.5)$ as the running buffer, separation voltage $-20 \mathrm{kV}$, detected at $200 \mathrm{~nm}$ and temperature controlled at $20^{\circ} \mathrm{C}$, a satisfactory separation of the six analytes was accomplished. The optimized method was validated for specificity, precision, linearity, accuracy and stability using sodium benzenesulfonate as the internal standard. The relative standard deviation for migration time was less than $0.58 \%$, and $3.78 \%$ for peak area ratio. The linearity ranged from 0.005 to $0.25 \mathrm{mM}$. The recovery ranged from $95.9 \%$ to $108.3 \%$. The limits of detection and limits of quantification for each enantiomer were 0.003 and $0.01 \mathrm{mM}$, respectively. This method was utilized for evaluating the chiral purity of five batches of Rotigotine.
\end{abstract}

(C) 2007 Elsevier B.V. All rights reserved.

Keywords: Capillary electrophoresis; Chiral separation; Dual cyclodextrin; Enantiomeric impurity; Pharmaceutical analysis; Rotigotine

\section{Introduction}

The pharmaceutical quality control is of great importance for maximizing the efficacy and the safety of the drug therapy and the economy of the production of drug [1]. Estimation of the impurities in pharmaceutical preparations is the most challenging and interesting work [2]. Pharmacological and toxicological characteristics often vary between enantiomers of the racemic drug because of the enantioselective biological inter-

\footnotetext{
* Corresponding author. Tel.: +86 1062792343; fax: +86 1062792343 .

** Corresponding author at: Department of Chemistry, Tsinghua University, Beijing 100084, China. Tel.: +86 1062792343; fax: +86 1062792343.

E-mail address: jmlin@mail.tsinghua.edu.cn (J.-M. Lin).
}

actions and reactions. In fact, one of them may be the most active enantiomer while the other may generate side-effects and/or toxicity. Thus, the determination of individual enantiomers is necessary for monitoring the pharmacokinetics and pharmacodynamics of chiral drugs. The separation and the quantification of enantiomeric mixtures were well studied in the past quarter-of-a-century in pharmaceutical and biomedical analysis and it still is an important area in pharmaceutical analysis.

Rotigotine, (-)-(S)-2-( $N$-propyl- $N$-2-thienylethylamino)-5hydroxytetralin, is a new non-ergolinic dopamine receptor agonist, being studied in the treatment of motor symptoms associated with Parkinson's disease (PD) and restless legs syndrome [3]. The chemical structures of Rotigotine, structurally related intermediates I (2-( $N$-propylamino)-5-hydroxytetralin) 

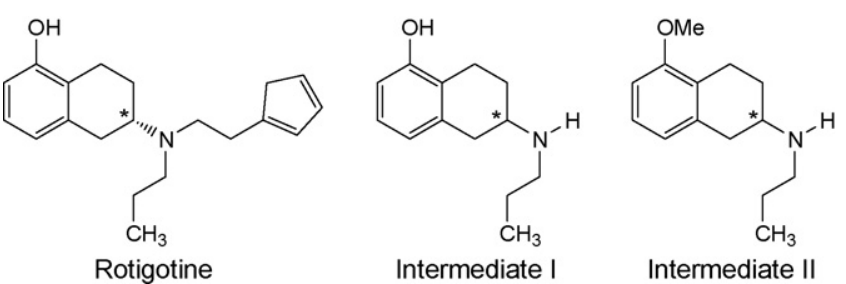

Fig. 1. Structures of the analytes investigated (* denotes an asymmetric carbon).

and II (2-( $N$-propylamino)-5-methoxytetralin) are shown in Fig. 1. Pharmacological studies have shown that Rotigotine is about 140 times more active than its $R$-enantiomer [4].

Capillary electrophoresis (CE) has been shown to be an effective technique in chiral separation due to its advantages, such as high resolution, short analysis time, flexibility, small sample volume requirement $(\mathrm{nL})$, low reagent consumption, etc. [5]. Since the introduction of the first commercially available instruments in the late 1980s, CE and related techniques, such as capillary electrochromatography (CEC), nonaqueous capillary electrophoresis (NACE), micellar electrokinetic chromatography (MEKC) and microemulsion electrokinetic chromatography (MEEKC) have attracted gradually increasing interest in chiral drug analysis as presented in many review papers [6,7]. Cyclodextrins (CDs) and their derivatives are the most widely used chiral selectors applied in chiral CE [8]. They have a shape of a truncated cone with a relatively hydrophobic cavity and a hydrophilic outside. The current mechanism for enantioseparation with a $C D$ involves inclusion complexation, and the primary interaction is hydrogen bond [9]. Charged CDs often have higher chiral resolving capacity than native CDs and they were introduced as chiral selectors in CE by Terabe [10]. With charged CDs, electrostatic forces play an important role in selector-selectand interactions. Therefore, chiral selectors carrying opposite charge to that of the selectands are very commonly utilized [7]. Several reviews have been published on the application of sulfated $\beta$-cyclodextrin (S- $\beta-C D$ ) to the chiral CE $[11,12]$.

The CD modified CE has been widely used for enantiomeric separation for a large number of compounds including many of pharmaceutical interest [13-15]. In some cases, the enantioseparation cannot be achieved by addition of one chiral selector to the running buffer. However, the dual CD system can often provide unique selectivities, facilitating or improving enantiomer separations. A dual CD system is usually a combination of a neutral and a charged $\mathrm{CD}$, or two charged CDs [8]. Dual CD systems were useful for chiral separation of basic drugs $[16,17]$.

To the best of our current knowledge, the CE method has not been previously employed for the simultaneous determination of Rotigotine, its $R$-enantiomer and the intermediates (I and II). The aim of this work, therefore, is to study the enantioseparation of these enantiomeric compounds by using the single $\mathrm{CD}$ system and the dual CD system.

\section{Experimental}

\subsection{Chemicals}

All reagents used in this study were of analytical grade, unless otherwise stated, and used without further purification. Rotigotine and its $R$-enantiomer ( $\mathrm{HCl}$ salts), the two intermediates I (HBr salts) and II ( $\mathrm{HCl}$ salts) (see Fig. 1) were synthesized and characterized by State Key Laboratory of Chemical Resource Engineering (Beijing University of Chemical Technology, Beijing, China). $\beta$-Cyclodextrin ( $\beta$-CD) was obtained from Nankai University Fine Chemical Lab (Tianjin, China). Sulfated $\beta$ cyclodextrin $(\mathrm{S}-\beta-\mathrm{CD}$, typical degree of substitution, 7-11) was obtained from Fluka (Schnelldorf, Germany). Methyl- $\beta$ cyclodextrin (M- $\beta-C D$, average degree of substitution, 12.5) was purchased from Wacker Fine Chemicals (Burghausen, Germany). Ultrapure water ( $>18 \mathrm{M} \Omega$ ) was obtained from an easypure $^{\mathrm{TM}}$ LF purification system with a $0.2 \mu \mathrm{m}$ fiber filter (Barnstead, USA).

\subsection{Instrumentation}

All experiments were carried out by a Beckman P/ACE ${ }^{\mathrm{TM}}$ MDQ Capillary Electrophoresis System (Fullerton, USA) equipped with a filter UV detector. The electropherograms were recorded and integrated by an IBM PC with $32 \mathrm{Karat}^{\mathrm{TM}}$ software version 4.0 (Beckman). Separation was performed in fusedsilica capillary (Yongnian Optical Fiber, Hebei, China) with a total length of $60 \mathrm{~cm}$ and an i.d. of $75 \mu \mathrm{m}$. A detection window was made at $50 \mathrm{~cm}$ from the inlet of the capillary by removing the polyimide coating. Direct UV detection was performed at the wavelength of $200 \mathrm{~nm}$. The power supply was operated in the constant-voltage mode.

\subsection{CE conditions}

A new capillary was initially washed with methanol for $10 \mathrm{~min}$, followed by ultrapure water for $5 \mathrm{~min}, 1 \mathrm{M} \mathrm{HCl}$ for $10 \mathrm{~min}$, ultrapure water for $5 \mathrm{~min}, 1 \mathrm{M} \mathrm{NaOH}$ for $10 \mathrm{~min}$, and ultrapure water for $5 \mathrm{~min}$. As a daily routine procedure, the capillary was rinsed with $1 \mathrm{M} \mathrm{NaOH}$ for $10 \mathrm{~min}$ followed by a 10-min rinse with ultrapure water, and then flushed with running buffer for $3 \mathrm{~min}$ before sample injections. Before each run, the capillary was rinsed with $1 \mathrm{M} \mathrm{NaOH}$ for $2 \mathrm{~min}$, ultrapure water for $2 \mathrm{~min}$, and running buffer for $2 \mathrm{~min}$. To achieve reproducible separations, all experiments were performed at $20 \pm 1{ }^{\circ} \mathrm{C}$ and were run in triplicate (or even more).

The background electrolyte (BGE) in the electrophoretic experiments was prepared by dissolving appropriate phosphate in ultrapure water and the BGE $\mathrm{pH}$ was adjusted with phosphoric acid $(1 \mathrm{M})$ or sodium hydroxide $(1 \mathrm{M})$. The chiral selectors were added to the BGE at the desired concentration. The running buffer was filtered with a $0.45 \mu \mathrm{m}$ filter before use. The injection was performed hydrodynamically by pressure at $0.3 \mathrm{psi}$ for $3 \mathrm{~s}$. Stock solution at the concentration of $10 \mathrm{mM}$ was prepared in ultrapure water, stored at $4{ }^{\circ} \mathrm{C}$ before use. Standard solutions of different concentrations were prepared by dilution of stock 
solution. The preparation of the real bulk sample was the same as the standard solution.

\subsection{Method validation}

The assessment of specificity, precision in migration time and peak area ratio, linearity, accuracy and stability of the drug was performed [18].

The precision of the method was determined as repeatability, intra-day and inter-day precision (intermediate precision) of migration times and peak area ratios. Peak area ratio is the ratio of the peak area of the analyte peak and the internal standard. Repeatability was determined by making six successive injections of a standard mixture solution at the concentration of $0.1 \mathrm{mM}$. The inter-day precision was evaluated over 3 days by performing six successive injections on each day. Accuracy of the method was established by performing recovery experiments at the concentration of $0.1 \mathrm{mM}$. Stability of the drug in solution was evaluated by injecting the sample to CE immediately after preparation and then reanalyzing after being stored on the bench-top for $24 \mathrm{~h}$.

\section{Results and discussion}

\subsection{Single CD system}

\subsubsection{Native $\beta-C D$}

$\beta-C D$ is the most widely used cyclodextrin due to its low cost and availability [8]. Thus, $\beta-C D$ was examined firstly as a chiral selector for Rotigotine and its $R$-form. According to the method development guideline for a basic compound [19], the buffer containing $50 \mathrm{mM}$ phosphate, $1.7 \%(\mathrm{w} / \mathrm{v}) \beta-\mathrm{CD}$ with normal polarity voltage $(+25 \mathrm{kV})$ was chosen as the initial experimental conditions. Then the influence of $\mathrm{pH}(2.5-12)$ of the BGE on the enantiomeric separation of the analytes was investigated. It was found that the best enantioseparation for Rotigotine and its $R$-form could be obtained at $\mathrm{pH} 6$ with the resolution of 2.5 (data not shown). Unfortunately, these $\mathrm{pH}$ variations did not benefit the chiral separation of the intermediates I and II. In order to improve the enantioseparation of the intermediates, the other parameters including $\beta-\mathrm{CD}$ concentration $(0.28-1.7 \%$, w/v), phosphate buffer concentration $(25-100 \mathrm{mM})$, organic modifier ( $\mathrm{MeOH}$, ethanol, isopropanol, $\mathrm{ACN}$, acetone, triethanolamine, trimethylamine, urea and triethylamine) have been explored. It was found that these parameters could not obviously improve the resolution of the intermediates I and II. This result is suggesting that the neutral $\beta-C D$ is not suitable to be the chiral selector for the basic enantiomeric compounds of the intermediates I and II.

From the results obtained above, it was also found that the enantiomeric peaks of Rotigotine and its $R$-form showed the tailing peaks (data not shown). This might be due to the electrostatic interaction between the positively charged analytes and the negatively charged capillary inner wall [20], or the difference in mobilities between the analyte ions and the buffer electrolyte ions [21]. The cetyltrimethylammonium chloride (CTAC) at the concentration of $150 \mu \mathrm{M}$ was added into the BGE, and it was found that it could successfully impair the peak tailing. CTAC contains a positively charged head group, thus it could interact with the dissociated silanol group by electrostatic interaction and create a cationic layer on the inner surface of the fusedsilica capillary [22]. This can prevent the interaction between the analytes and the capillary wall.

\subsubsection{Sulfated $\beta-C D$}

As discussed in the previous section, the native $\beta-C D$ could not achieve the enantioseparation of the intermediates I and II. Therefore, the charged sulfated $\beta-C D(S-\beta-C D)$ was chosen and examined, considering the basic nature of these compounds. The BGE containing $50 \mathrm{mM}$ phosphate buffer, $4 \%$ (w/v) S- $\beta$ $\mathrm{CD}$ was preliminarily employed, and this solution was finally adjusted to $\mathrm{pH} 2.5$. The analytes were positively charged at $\mathrm{pH}$ 2.5 and they would migrate to the cathode in the separation buffer without $S-\beta-C D$ under the normal polarity. The addition of S- $\beta-C D$ made the direction of the effective mobility opposite to the electroosmotic flow (EOF) because of the interaction between the analytes and the negatively charged chiral selector. Considering the low EOF at $\mathrm{pH} 2.5$, the reversed polarity $(-20 \mathrm{kV})$ was applied to ensure the detection at the outlet end of the capillary. The obtained results are shown in Fig. 2. Rotigotine and its $R$-form, and the intermediate II enantiomers were baseline enantioseparated, meanwhile the intermediate I enantiomers were partially separated. Unfortunately, the migration times of Rotigotine and its $R$-form were very similar to those of the intermediate II enantiomers. However, these results have suggested that $S-\beta-C D$ showed higher chiral separation capacity compared with $\beta-C D$. It might be attributed to the sulfate groups of the $S-\beta-$ CD randomly distributed on the narrow end of the torus as well as along the wide rim of the CD cavity [23]. The surrounding sulfate moieties may assist in immobilizing the analyte in a way that encourages complexation and facilitates the enantiospecific interactions by strong coulombic interaction with basic drugs, thus enhance the chiral discrimination capacity [24,25].

In addition, the $\mathrm{pH}$ variation has been studied to alter the enantioselectivity of Rotigotine and its $R$-form, and the intermediate II enantiomers. The $\mathrm{pH}$ ranging from the acidic $\mathrm{pH}$ to the neutral $\mathrm{pH}$ was considered to maintain the same CE separation mechanisms. The results were found that Rotigotine and its $R$-form, and the intermediate II enantiomers could be enantioresolved at $\mathrm{pH}$

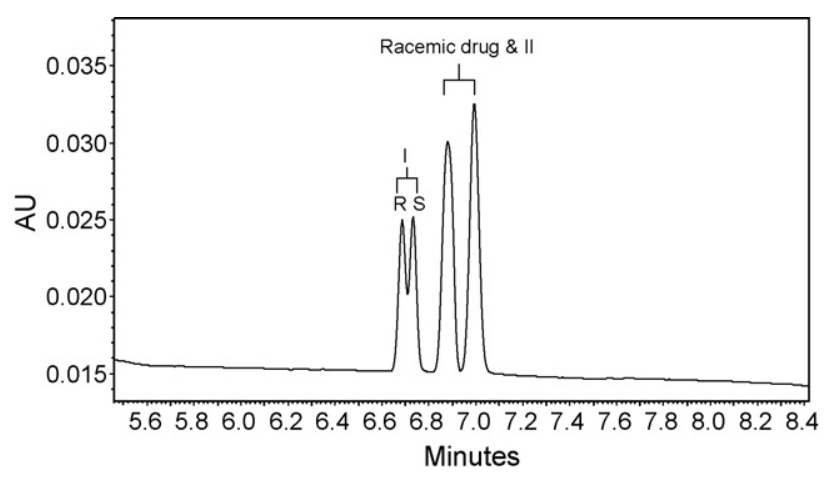

Fig. 2. Electropherogram of Rotigotine, its enantiomer and intermediates (I and II) using S- $\beta-\mathrm{CD}$ as chiral selector. Conditions: $50 \mathrm{mM}$ phosphate, $4 \%(\mathrm{w} / \mathrm{v})$ $\mathrm{S}-\beta-\mathrm{CD}, \mathrm{pH} 2.5$, running voltage $-20 \mathrm{kV}$. 


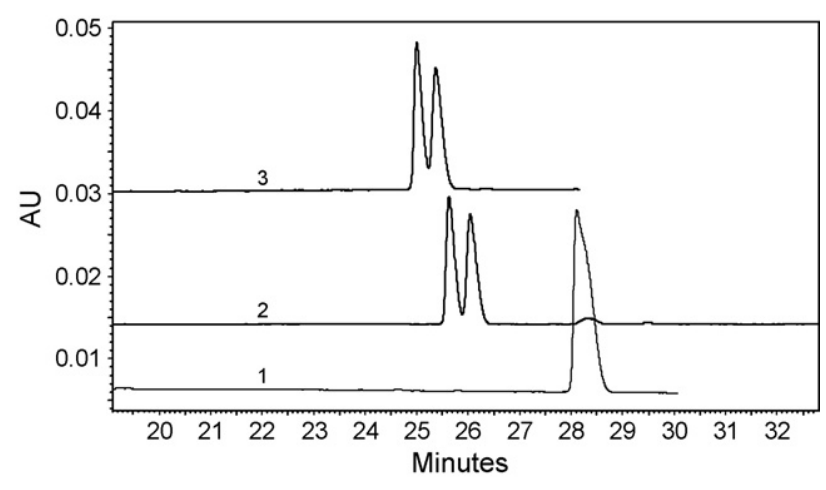

Fig. 3. Electropherograms of (1) Rotigotine and its enantiomer; (2) intermediate $\mathrm{I}$; (3) intermediate II. Conditions: $50 \mathrm{mM}$ phosphate, $2 \%$ (w/v) M- $\beta-\mathrm{CD}, \mathrm{pH} 2.5$, normal polarity $+20 \mathrm{kV}$.

5.5 with significant difference in migration times. Meanwhile, the intermediate I could not be enantioseparated. Although the $\mathrm{pH} 5.5$ seemed provide the greater enantioselectivtiy than that of $\mathrm{pH} 2.5$, the $\mathrm{pH} 2.5$ was chosen and used further. Because the low $\mathrm{pH}$ (2.5) provided the partial enantioseparation for the intermediate I and effectively suppressed the dissociation of the silanol on the capillary inner wall and prevented interactions between the analytes and the capillary wall, thereby eliminating the peak tailing.

\subsubsection{Methyl- $\beta-C D$}

Another neutral derivative $C D$ was also investigated. The methyl- $\beta-C D(M-\beta-C D)$ was chosen and studied in this section. The BGE containing $50 \mathrm{mM}$ phosphate buffer, $2 \%(\mathrm{w} / \mathrm{v})$ $\mathrm{M}-\beta-\mathrm{CD}$ was preliminarily employed. The $\mathrm{pH}$ of the BGE was finally adjusted to 2.5 and the applied voltage was $+20 \mathrm{kV}$. The results are shown in Fig. 3. It was found that the intermediate enantiomers (I and II) could be partially enantioseparated with the resolutions higher than 1.1. Meanwhile, the Rotigotine and its $R$-form could not be enantioseparated. These results are suggesting that the M- $\beta-C D$ showed high enantioselectivity to chiral intermediates (I and II). To explain this result, the molecular structures of the studied analytes should be taken into account. The intermediates I and II were less hydrophobic than the chiral drug (Rotigotine and its $R$-form), because the chiral drug contains the thiophen group in its structure. On the other hand, the methyl group of the M- $\beta-\mathrm{CD}$ can provide more hydrophobicity for $\mathrm{CD}$ cavity. The more hydrophobic cavity might enhance the host-guest interactions between the hydrophobic selectand and hydrophobic selector [26]. This hypothesis could be confirmed by the longer migration times of racemic drugs (Rotigotine and its $R$-form) compared with the intermediates.

Moreover, the effect of $\mathrm{pH}(2.5-7.0)$ on the enantioselectivity of M- $\beta-\mathrm{CD}$ for the studied analytes has been investigated. But these variations did not significantly affect the chiral recognition. Therefore, $\mathrm{pH} 2.5$ was selected and used further.

\subsection{Dual CD system}

Based on the above results, S- $\beta$-CD provided the good enantioseparation for chiral drug and the intermediate II, mean- while M- $\beta$-CD provided the good enantioseparation for the intermediates (I and II). Hence, a dual CD system containing S- $\beta-C D$ and $M-\beta-C D$ was proposed. Considering the good performance of each single $\mathrm{CD}$ system at $\mathrm{pH} 2.5$, therefore, the $\mathrm{pH}$ of the BGE for the dual CD system used in this section was also simply selected at 2.5. The BGE containing $50 \mathrm{mM}$ phosphate buffer, $3 \%(\mathrm{w} / \mathrm{v}) \mathrm{S}-\beta-\mathrm{CD}$ and $2 \%(\mathrm{w} / \mathrm{v}) \mathrm{M}-$ $\beta$-CD was preliminarily examined. The separation voltage was $-20 \mathrm{kV}$ and the detection wavelength was set at $200 \mathrm{~nm}$. As expected, it was found that all enantiomers were enantioseparated well without peak tailing, especially for Rotigotine and its $R$-form, and the intermediate II (data not shown). Under this CE system, the intermediate I could be partially enantioseparated $\left(R_{\mathrm{S}}=0.9\right)$.

The effect of the concentration of S- $\beta-C D$ and $M-\beta-C D$ on the enantioseparation was also investigated. The concentration of $S-\beta-C D$ and $M-\beta-C D$ was univariated ranging from 1 to $6 \%$ $(w / v)$, and vice versa. The migration times increased with the increase of the M- $\beta-C D$ concentration because the buffer viscosity was increased. The increase of S- $\beta-C D$ concentration generated higher current and shortened the migration times. The higher current was attributed to the increased number of the charged chiral selectors in the buffer. The higher S- $\beta-C D$ concentration resulted in more complex of the analytes and the chiral selector, which increased the apparent mobility. The data showed that the optimal concentration for both S- $\beta-C D$ and M$\beta-\mathrm{CD}$ was $2 \%(\mathrm{w} / \mathrm{v})$, taking the resolution and migration times into consideration. Under this condition, the enantiomers of I could be partially enantioseparated $\left(R_{\mathrm{S}}=0.8\right)$, and the other four isomers could be completely enantioseparated (data not shown).

In addition, the influence of the ionic strength of the BGE on the enantioselectivity was studied. The ionic strength variation was evaluated by varying the phosphate concentration from 10 to $100 \mathrm{mM}$. Increasing ionic strength resulted in the reduction of EOF and the increment of buffer viscosity. The resolution of chiral compounds, especially for intermediate I, increased with increasing concentration of phosphate buffer. It was eventually found that $100 \mathrm{mM}$ phosphate was the optimal concentration for this dual CD system, providing a short analysis time less

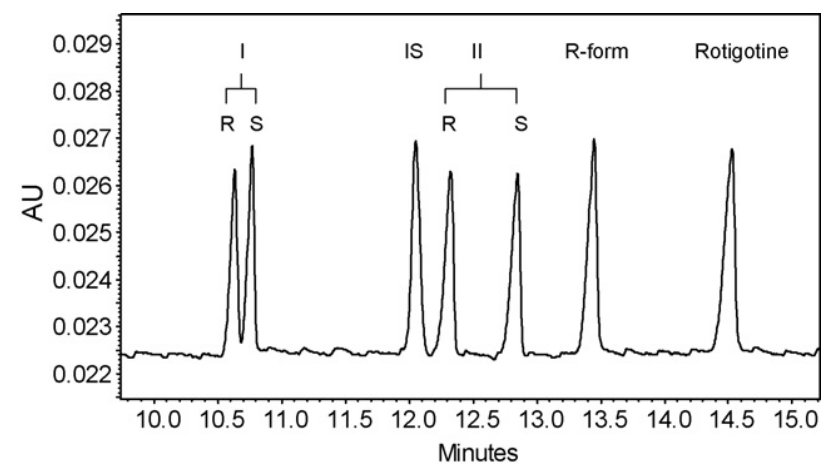

Fig. 4. Electropherogram of the simultaneous enantioseparation of three chiral compounds, including Rotigotine, its enantiomer, and intermediates I and II. Conditions: $100 \mathrm{mM}$ phosphate, $2 \%$ (w/v) S- $\beta-\mathrm{CD}, 2 \%$ (w/v) M- $\beta-\mathrm{CD}, \mathrm{pH} 2.5$, $-20 \mathrm{kV}$. The concentrations of all analytes were $0.05 \mathrm{mM}$. 
Table 1

Enantioresolution capacity of the single $\mathrm{CD}$ and the dual $\mathrm{CD}$ system

\begin{tabular}{|c|c|c|c|c|}
\hline \multirow[t]{2}{*}{ Selector } & \multirow[t]{2}{*}{ Compound } & \multicolumn{2}{|c|}{ Migration time (min) } & \multirow[t]{2}{*}{ Resolutior } \\
\hline & & $t_{\mathrm{R}}$ & $t_{\mathrm{S}}$ & \\
\hline \multirow[t]{2}{*}{$4 \% \mathrm{~S}-\beta-\mathrm{CD}^{\mathrm{a}}$} & Intermediate I & 6.667 & 6.713 & 0.7 \\
\hline & Racemic drug & 6.862 & 6.992 & 1.8 \\
\hline \multirow[t]{2}{*}{$2 \% \mathrm{M}-\beta-\mathrm{CD}^{\mathrm{b}}$} & Intermediate I & 25.58 & 25.99 & 1.2 \\
\hline & Intermediate II & 25.00 & 25.37 & 1.1 \\
\hline \multirow{3}{*}{$\begin{array}{l}\text { Dual CD system }{ }^{\mathrm{c}} 2 \% \\
\quad \text { S- } \beta-C D+2 \% \mathrm{M}-\beta-\mathrm{CD}\end{array}$} & Intermediate I & 10.658 & 10.801 & 1.3 \\
\hline & Intermediate II & 12.376 & 12.907 & 4.1 \\
\hline & Racemic drug & 13.512 & 14.610 & 7.3 \\
\hline
\end{tabular}

a $50 \mathrm{mM}$ phosphate, $\mathrm{pH} 2.5,-20 \mathrm{kV}$.

b $50 \mathrm{mM}$ phosphate, $\mathrm{pH} 2.5,+20 \mathrm{kV}$.

c $100 \mathrm{mM}$ phosphate, $\mathrm{pH} 2.5,-20 \mathrm{kV}$.

than $15 \mathrm{~min}$. The typical electropherogram of the studied analytes is shown in Fig. 4. Moreover, the results shown in Table 1 could obviously describe the synergistic effects of the dual $\mathrm{CD}$ system on the successful enantioseparation of the studied analytes.

\subsection{Method validation}

The optimized method containing dual CD system was validated for quantification of the chiral drug using sodium benzenesulfonate as the internal standard. Sodium benzenesulfonate was used because it provided stable detector response and its migration time was close to those of the analytes (see Fig. 4). The validation of the method was evaluated by means of the specificity, precision, linearity, sensitivity and accuracy. The obtained results are shown in Tables 2 and 3.

In the present method, acceptable resolution of enantiomers of the racemic drug and its chiral intermediates I and II was achieved. In applications for drug marketing authorization for single enantiomeric compounds there is a need for relatively rapid, precise and sensitive enantioselective methods which should allow detection of the trace enantiomer at sub $1 \%(\mathrm{w} / \mathrm{w})$ concentration. The separation obtained of the pure Rotigo-

Table 2

Precision data

\begin{tabular}{ll}
\hline & R.S.D. $(\%)$ \\
\hline Repeatability $^{\mathrm{a}}$ & \\
$\quad$ Migration time & 0.07 \\
Peak area ratio & 0.78 \\
Intra-day precision $^{\mathrm{b}}$ & \\
$\quad$ Migration time $^{\text {Peak area ratio }}$ & 0.24 \\
Inter-day precision $^{\mathrm{c}}$ & 3.78 \\
$\quad$ Migration time & \\
Peak area ratio & 0.58 \\
\hline
\end{tabular}

\footnotetext{
${ }^{\text {a }}$ Six successive injections.

b Six successive injections at different time within the same day.

${ }^{c}$ Six successive injections per day, evaluated over 3 days.
}

tine spiked of $0.1 \%$ (w/w) $R$-enantiomer was shown in Fig. 5 . In all the cases the relative standard deviation (R.S.D.) was less than $0.58 \%$ in migration time and $3.78 \%$ in peak area ratio. The linearity ranged from 0.005 to $0.25 \mathrm{mM}$ and the correlation coefficients $(R)$ of the analytes were from 0.9978 to 0.9995 . The LOD and LOQ for each enantiomer were found to be 0.003 and $0.01 \mathrm{mM}$, respectively. The recovery ranged from $95.9 \%$ to $108.3 \%$. No significant change was observed in the peak area ratios confirming that no degradation or interconversion of the enantiomers took place for at least $24 \mathrm{~h}$.

These results showed this CE method was reliable for the determination of Rotigotine and the related impurities. Therefore, these good characteristics allowed the applicability of the method to the simultaneous determinations of Rotigotine and the impurities in real samples.

\subsection{Application in bulk samples}

Five various batches of Rotigotine were analyzed for the impurity contents. Lot 1 contained $24.13 \%$ impurities in all due to the incomplete reaction and the lack of recrystallization during the preparation process of this batch. The electropherogram of Lot 1 is shown in Fig. 6. No impurity was detected in Lots 2-4. Lot 5 was a product without chemical indirect chiral sepa-

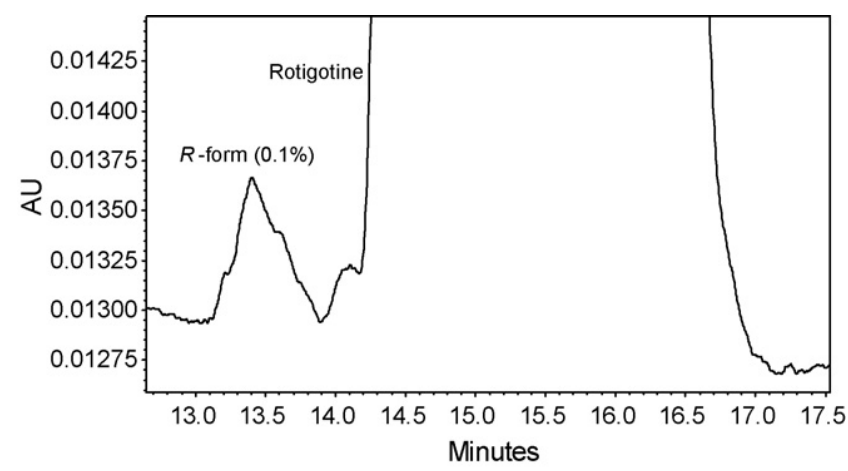

Fig. 5. Analysis of trace $(0.1 \%$, w/w) enantiomeric impurity in Rotigotine (12.5 mM). Conditions used as in Fig. 4. 
Table 3

Characteristics of the regression lines, accuracy and recovery

\begin{tabular}{|c|c|c|c|c|c|c|c|}
\hline \multirow[t]{2}{*}{ Analyte } & & \multicolumn{3}{|c|}{ Regression line } & \multirow[t]{2}{*}{ LOD (mM) } & \multirow[t]{2}{*}{ LOQ (mM) } & \multirow[t]{2}{*}{ Recovery (\%) } \\
\hline & & $a$ & $b$ & $R$ & & & \\
\hline \multirow{3}{*}{ II } & $R$ & 17.9 & -0.0934 & 0.9978 & \multirow{4}{*}{0.003} & \multirow{4}{*}{0.01} & 103.19 \\
\hline & $R$ & 19.8 & -0.0619 & 0.9986 & & & 95.93 \\
\hline & $S$ & 21.6 & -0.1065 & 0.9978 & & & 96.62 \\
\hline$R$-form & & 25.5 & -0.1054 & 0.9982 & & & 99.16 \\
\hline
\end{tabular}

Calibration curves are expressed as regression lines $(y=a x+b)$, where $y$ is the peak area ratio of the analyte to the internal standard, $x$ is analyte concentration (mM), $a$ is slope, $b$ is intercept, and $R$ is correlation coefficient, $n=3$ determinations.

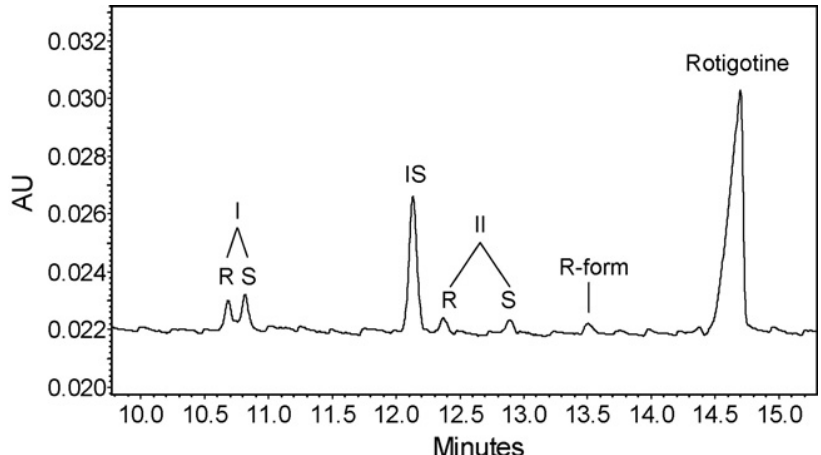

Fig. 6. Electropherogram of the real Rotigotine product (Lot 1, $0.1 \mathrm{mM}$ ). Conditions used as in Fig. 4.

ration, thus this batch was a racemate and it contained 50.29\% $R$-enantiomer of Rotigotine.

\section{Conclusions}

The three single CD systems were not successful in separating Rotigotine and related impurities simultaneously. Then a dual CD system containing S- $\beta-C D$ and $M-\beta-C D$ was demonstrated. It was an effective chiral CE method to enantioresolve and determine Rotigotine and its chiral impurities within $15 \mathrm{~min}$. The optimized method was highly specific, sensitive, accurate and reproducible. And it was also applied to estimate the impurities of five batches of Rotigotine.

\section{Acknowledgements}

This work was supported by the National Natural Science Foundation of China (Grant Nos. 20575008, 20437020) and the Program for Changjiang Scholars and Innovative Research Team in University (No. IRT0404).

\section{References}

[1] S. Görög, Trends Anal. Chem. 26 (2007) 12-17.

[2] S. Görög, Trends Anal. Chem. 25 (2006) 755-757.

[3] V. Bertaina-Anglade, C.D. La Rochelle, D.K.A. Scheller, Eur. J. Pharmacol. 548 (2006) 106-114.

[4] Manimaran, Thanikavelu, Impastato, J. Fred, US Patent 4,968,837, (6 November 1990).

[5] B. Chankvetadze, Capillary Electrophoresis in Chiral Analysis, Wiley, New York, 1997.

[6] G.K.E. Scriba, J. Pharm. Biomed. Anal. 27 (2002) 373-399.

[7] P.T.T. Ha, J. Hoogmartens, A. Van Schepdael, J. Pharm. Biomed. Anal. 41 (2006) $1-11$

[8] M. Blanco, I. Valverde, Trends Anal. Chem. 22 (2006) 428-429.

[9] A. Berthod, Anal. Chem. 78 (2006) 2093-2099.

[10] S. Terabe, Trends Anal. Chem. 8 (1989) 129-134.

[11] C.E. Evans, A.M. Stalcup, Chirality 15 (2003) 709-723.

[12] T.J. Ward, Anal. Chem. 78 (2006) 3947-3956.

[13] G. Blaschke, B. Chankvetadze, J. Chromatogr. A 875 (2000) 3-25

[14] K.D. Altria, D. Elder, J. Chromatogr. A 1023 (2004) 1-14.

[15] K.D. Altria, A. Marsh, C. Sänger-van de Griend, Electrophoresis 27 (2006) 2263-2282.

[16] S. Izumoto, H. Nishi, Electrophoresis 20 (1999) 189-197.

[17] M. Fillet, Ph. Hubert, J. Crommen, J. Chromatogr. A 875 (2000) 123-134.

[18] International Conference on Harmonisation, ICH Harmonised Tripartite Guideline, Validation of Analytical Procedures: text and methodology Q2(R1), International Conference on Harmonisation of Technical Requirements for Registration of Pharmaceuticals for Human Use, November, 2005 (http://www.ich.org/cache/compo/276-254-1.html).

[19] K.D. Altria, Capillary Electrophoresis Guidebook: Principles, Operation, and Applications, Humana Press, New Jersey, 1996.

[20] K.D. Altria, J. Pharm. Biomed. Anal. 31 (2003) 447-453.

[21] P. Gebauer, P. Boček, Anal. Chem. 69 (1997) 1557-1563.

[22] J. Varghese, R.B. Cole, J. Chromatogr. A 652 (1993) 369-376.

[23] K.W. Phinney, J.W. Jackson, L.C. Sander, Electrophoresis 23 (2002) 1308-1313.

[24] F.-T.A. Chen, G. Shen, R.A. Evangelista, J. Chromatogr. A 924 (2001) 523-532.

[25] V. Maier, J. Horáková, J. Petr, E. Tesařová, P. Coufal, J. Ševčík, J. Pharm. Biomed. Anal. 39 (2005) 691-696.

[26] S. Fanali, J. Chromatogr. A 735 (1996) 77-121. 